\title{
ATYPICAL PRESENTATION OF PERIVENTRICULAR HAEMORRHAGIC INFARCTION IN THE PRESENCE OF THROMBOPHILIA
}

\author{
J. Harteman ${ }^{1}$, F. Groenendaal ${ }^{1}$, D. Liem ${ }^{2}$, H. Stroink ${ }^{3}$, M. Bierings ${ }^{4}$, L. de Vries ${ }^{1}$ \\ ${ }^{I}$ Department of Neonatology, Wilhelmina Children's Hospital, University Medical Center, Utrecht, \\ ${ }^{2}$ Department of Neonatology, Radboud University Nijmegen Medical Center, ${ }^{3}$ Department of Neurology, \\ Canisius Wilhelmina Hospital, Nijmegen, ${ }^{4}$ Department of Paediatric Haematology \& Oncology, Wilhelmina \\ Children's Hospital, University Medical Center, Utrecht, The Netherlands
}

Introduction: Periventricular haemorrhagic infarction (PVHI) is a complication of preterm birth associated with cardiorespiratory instability. The role of thrombophilia as a possible additional risk factor in infants with an atypical presentation when PVHI was of presumed antenatal onset or $>96 \mathrm{~h}$ after birth in the absence of a preceding sudden deterioration, has not been investigated extensively.

Objective: To report thrombophilic mutations in preterm infants with an atypical presentation of PVHI.

Methods: From January 2005 - December 2010 data of infants (gestational age 28.7 to 36.7 weeks, median 30.6 weeks; birth weight 580 to 3090 gram, median 1300 gram) who developed an atypical PVHI were collected. Mutation analysis of the factor V Leiden (G1691A), prothrombin (G20210A) gene and C677T and A1298C polymorphisms in the MTHFR gene was performed, as well as the plasma lipoprotein(a) and homocysteine levels.

Results: Twenty-one preterm infants presented with an atypical PVHI. Among these infants a heterozygous factor V Leiden mutation was found in seven (33\%) infants and their mothers (population incidence is 3$5 \%$ ), in one concomitant with a heterozygous prothrombin gene mutation and one had an isolated heterozygous prothrombin gene mutation. C677T or A1298C polymorphism in the MTHFR gene was present in all these 8 infants. In two siblings, a mutation in COL $4 A 1$ gene was found.

Conclusions: An atypical presentation of PVHI in preterm infants is often associated with gene mutations, especially the factor V Leiden mutation. 